\title{
Relevance of Bell Inequalities For Interpreting EPR-Bohm Experiments
}

\section{E. A. Scarzafava}

(Dated: October 18, 2020)

\begin{abstract}
The various Bell Inequalities used to interpret the results of EPR-Bohm experiments are shown to be inappropriate for such experiments. The inequalities do not allow for correlation between the particles, which is the basis for such experiments, and therefore are irrelevant. Claims that the results of such experiments, because they violate the inequalities, require the conclusion that the measurement of one particle in an "entangled" pair can instantly affect the state of its partner, even at great distances, are untrue and need to be modified.
\end{abstract}

\section{BACKGROUND}

In 1935 Einstein, Poldolsky and Rosen[1] (EPR) pre- ${ }^{57}$ sented a paper in which they concluded that the quan- ${ }^{58}$ tum mechanical description of reality given by wave me- ${ }^{59}$ chanics is not complete. They did this by first showing ${ }^{60}$ that either (1) the wave function description is not com- ${ }^{61}$ plete or (2) physical quantities corresponding to two non- ${ }^{62}$ commuting operators cannot have simultaneous reality. ${ }^{63}$ They described a thought experiment in which two sys- ${ }^{64}$ tems start out with interactions between them, but sub- ${ }^{65}$ sequently can no longer interact. Then, starting with the ${ }^{66}$ assumption that the wave function does give a complete ${ }^{67}$ description, they showed that the two physical quanti- ${ }^{68}$ ties of non-commuting operators can have simultaneous ${ }^{69}$ reality. The negation of alternative (1) leads to the nega- ${ }^{70}$ tion of alternative (2), and thus their stated conclusion. ${ }^{71}$ They stressed the fact that by simultaneously real they ${ }^{72}$ are referring to quantities that either one or the other, ${ }^{73}$ but not not both simultaneously, of the two quantities ${ }^{74}$ can be predicted. They expressly exclude the case where ${ }^{75}$ both quantities can be simultaneously measured or predicted, because that would make the reality of the physical quantities "depend upon the process of measurement ${ }^{76}$ carried out on the first system, which does not disturb the second system in any way."

It is difficult to carry out such experiments, but in ${ }^{78}$ 1957 Bohm and Aharonov[2] described experiments that ${ }^{79}$ can be performed that test the concept of the EPR para- ${ }^{80}$ dox. In one case, two electrons in a singlet spin state ${ }^{81}$ are separated by a method that does not alter the total ${ }^{82}$ spin and, when they are sufficiently far apart, measure- ${ }^{83}$ ments are made on the two systems. In another case, ${ }^{84}$ two photons are created with opposite momentum and ${ }^{85}$ with each photon in a polarization state that is orthog- ${ }^{86}$ onal to the other. The experiments were still difficult ${ }^{87}$ to carry out with the technology available at that time, ${ }^{88}$ but preliminary results appeared to favor the view that ${ }^{89}$ measurements on one system affected measurements on ${ }^{90}$ the correlated system that was spatially distant from the ${ }^{91}$ first. They did allow for the possibility that quantum ${ }^{92}$ theory is not complete and may be an approximation ${ }_{93}$ arising from "a deeper subquantum-mechanical level". $\quad 94$

In 1964 Bell[3] derived a set of inequalities that he 95 claimed must apply to any system, even with hidden vari- 96 ables, if that system were to meet the requirements of lo- 97 cality (i.e., measurements on one system can not affect, nor be affected by measurements on the other). Using the example of of an experiment based on the BohmAharonov experiment[2] (EPR-Bohm experiments), and imposing his locality requirement, Bell developed inequalities that were incompatible with the statistical predictions of quantum mechanics.

Subsequent experiments, such as those using an optical analog of the Stern-Gerlach filters of Bell's paper, have shown dramatic violations of variations of Bell's inequalities, while being in good agreement with quantum mechanical predictions.[4] This has led to the conclusion that experiments show that a measurement on either member of an "entangled" pair has an instantaneous effect on on the state of the other, regardless of the distance between them (spooky action-at-a-distance). That view is reflected in college textbooks and material intended to educate and inform the general public.[5] In the next section we show that view to be incorrect, because inappropriate localization requirements were used in the development of the inequalities.

\section{CRITIQUE OF THE BELL INEQUALITIES}

In this paper it is shown that violation of the Bell Inequalities does not prove non-local, action at a distance behavior for "entangled" particles. The criteria used to impose locality in the development of the inequalities make them meaningless in the interpretation of the experimental results of EPR-Bohm experiments. There are several variations of inequalities used to test the EPR-Bohm experiments, all of which are referred to generically as Bell Inequalities. In this section it is shown that they all use localization criteria that are incorrect for such experiments.

In 1978 Clauser and Shimony[6] provided an excellent review of the then current Bell Inequalities. This paper relies heavily on their review. In Section 3.3 of Clauser and Shimony they discuss the locality concept and provide a very reasonable definition:

Suppose a pair of correlated systems, which have a joint state $\lambda$, separate. They then continue to evolve perhaps in an inherently stochastic way, and given $\lambda$, a and b, one can define probabilities for any particular out- 
come at either apparatus. We allow that, given $\lambda$, these two probabilities may each depend on the associated (local) apparatus parameter, a or b respectively, and of course upon $\lambda$, but we assume that these probabilities are otherwise independent of each other.

This definition of locality seems very common-sensical. It says that the outcome (or the probability of outcomes) of a measurement performed on one part of a composite system is independent of what aspects of the other component the experimenter chooses to measure. It by no means excludes the possibility of obtaining knowledge concerning system 2 from an examination of system 1 . The state $\lambda$ contains information common to both systems, and a measurement on one of these presumably reveals some of this. Nor does it prevent a measurement performed on one component of a composite system from locally disturbing that component. What it does prescribe, in essence, is that the measured value of a quantity on one system is not causally affected by what one chooses to measure on the other system, since the two systems are well separated (e.g. spacelike separated) when the measurements are performed.[6]

It is difficult to design experiments to test the original Bell Inequalities; so experiments have been designed to be interpreted by variations of the inequalities, many of which are discussed in the following subsections.

\section{A. Clauser and Horne}

We start with Clauser and Horne[7]. This is because ittr3 most simply demonstrates the localization problem with ${ }^{174}$ Bell Inequalities and because Clauser-Horne $(\mathrm{CH})$ type ${ }^{175}$ Bell Inequalities are the ones commonly used for EPR-176 Bohm experiments. In this description of their develop-177 ment many of the details and explanations are glossed over, but much of it is straight from their paper.

Clauser and Horne, describe a typical EPR-Bohm experiment, using an experimental arrangement in which $\mathrm{a}^{178}$ source of coincident two-particle emissions is viewed by ${ }^{179}$ two analyzer-detector assemblies, 1 and 2. Each apparatus has an adjustable parameter, a at apparatus 1 and b at apparatus 2, where a and $\mathrm{b}$ are angles specifying the orientation of the analyzers, e.g., the axes of linear polar-180 izers for photons or the directions of the field gradients ${ }_{181}$ of Stern-Gerlach magnets for fermions.

During a period of time, while the adjustable param-183 eters have the values a and $\mathrm{b}$, the source may emit $\mathrm{N}$ of $f_{184}$ the two-particle systems of interest. Let $N_{1}(a)$ and $N_{2}(b)_{185}$ be the number of counts at at detectors 1 and 2, respec-186 tively, and let $N_{12}(a, b)$ be the number of simultaneous187 counts from the two detectors (coincident counts). When $\mathrm{N}$ is sufficiently large the ensemble probabilities are given by Clauser and Horne's Equations (1):

$$
p_{1}(a)=N_{1}(a) / N
$$

155

156

where $\Gamma$ is the space of the states $\lambda$.

$* * * * * * * * * * * * * * * * * * * * * * * * * * * * * * * * * * * * * * * * * * * * * * * * * * * * * * * *$

This is where the crucial error in their development is made. In the equation for the ensemble probability for the coincident counts they make the substitution, their Equation $\left(2^{\prime}\right)$

$$
p_{12}(\lambda, a, b)=p_{1}(\lambda, a) p_{2}(\lambda, b)
$$

and they justify this as a reasonable locality condition, which leads to

$$
p_{12}(a, b)=\int_{\Gamma} d \lambda \rho(\lambda) p_{1}(\lambda, a) p_{2}(\lambda, b)
$$

However, this factorization is definitely not a reasonable locality condition. It is an unacceptable one, because it assumes that there is no correlation between the two particles that were emitted. It defeats the whole purpose of the EPR-Bohm experiments. A great deal of time, effort and ingenuity is expended by the experimenters to construct the sophisticated apparatus used to 
create correlated particle pairs and to ensure that their ${ }_{242}$ spin or polarization states are not disturbed by external means until they encounter the detectors. EPR[1] in their thought experiment stake their claim of simultaneous reality on the ability to obtain information about one system from measurements made on the other be- ${ }^{243}$ cause of the correlation between the two systems. If $^{244}$ there were no correlations between the particles at the $\mathrm{e}^{245}$ time that the measurements are made, then their thought ${ }^{246}$ experiment would indeed fail. Quantum mechanics also ${ }^{247}$ predicts that there are correlations between the systems. ${ }^{248}$ Fortunately, the results of the experiments that are able ${ }^{249}$ to be performed[4] indicate that both EPR and quantum ${ }^{250}$ mechanics are correct about that. EPR did not claim ${ }^{251}$ that quantum mechanics was wrong, just that its descrip- ${ }^{252}$ tion was incomplete.

Note: It could be said that correlation between the particles is included in Equation (2) through the use of $\lambda$, which can represent shared variables. Each particle shares a common set of values or instructions and thus two particles effectively are correlated when each separately interacts with its detector. This position fails on several levels. (I) The correlations associated with the singlet state are specific for each separate pair of particles. The sum of the pairs of correlated values is not equal to the product of the sums of the individual values. (II) No use is made of any potential correlation in deriving the inequalities. (III) The correlation of interest is the fact that the spins or polarizations of the particles are complementary, because they were created in a singlet state. Hidden variables may explain why each particle exhibits a certain value when measured, but are not the source of the correlation of interest. (IV) This factored probability is equivalent to deterministic models, such as those discussed in Section II.B, below, which make no use of hidden variables and yield precisely the same inequalities. Disjoint subspaces do not provide for complementarity in the measurements for each pair.

Clauser and Shimony $[6]$ in their Section 3.6 and Equations (3.21) assume from symmetry considerations that

$$
\begin{aligned}
& p_{1}(a) \text { is independent of } \mathrm{b} \\
& p_{2}(b) \text { is independent of a } \\
& \text { and } p_{12}(a, b) \equiv p_{12}(|a-b|)
\end{aligned}
$$

That is a much more reasonable expression for $p_{12}(a, b)_{284}$ than Equation (2) above, because it implies correlation 285 between the particles. The correlated pair of particles was created in a singlet state. The spin/polarization state for the correlated system can be represented by the wave function

$$
\Psi=\frac{1}{\sqrt{2}}\left[\psi_{+}(1) \psi_{-}(2)-\psi_{-}(1) \psi_{+}(2)\right]
$$

This wave function describes the complementary relationship between the spins of the two particles. If the particles experience no interactions to disturb the spin state of either particle, that relationship should not change. Thus, because the particles are complementary, a measurement on one particle can provide information on the other at the time of the measurement, regardless of how far apart they are. EPR's claim for simultaneous reality depends on that relationship. Quantum mechanics also supports that view, at least for a large enough ensemble of particles. The disagreement between Einstein and Bohr is whether that relationship implies simultaneous reality (Einstein) or that the spin state for each particle only obtains reality when one of them is disturbed and the wave function collapses (Bohr).

[Note: Some would criticize this description as being too classical, because in quantum theory only one component of the spin of each particle can have a definite value at a given time. Quantum theory also says that no matter which component of the spin of particle 1 is measured at detector a, the same component of the spin of particle 2 will have a definite and opposite value when the measurement is over. But we are free to choose any direction as the one in which the spin of particle 1 (and therefore of particle 2) will become definite; and therefore a measurement at one detector can influence the measurement at the other.][2]

The problem with that criticism is that it presupposes that EPR are wrong when they assert simultaneous reality for the spins of the particles. The purpose of an EPR-Bohm experiment is to test that very issue.

However, Clauser and Horne use Equation (2), which denies any correlation between the particles at the time of the measurement, to obtain their inequality. They prove a mathematical lemma and, using that lemma, establish the inequality

$$
\begin{array}{r}
-1 \leq p_{1}(\lambda, a) p_{2}(\lambda, b)-p_{1}(\lambda, a) p_{2}\left(\lambda, b^{\prime}\right)+p_{1}\left(\lambda, a^{\prime}\right) p_{2}(\lambda, b) \\
+p_{1}\left(\lambda, a^{\prime}\right) p_{2}\left(\lambda, b^{\prime}\right)-p_{1}\left(\lambda, a^{\prime}\right)-p_{2}(\lambda, b) \leq 0
\end{array}
$$

where $a$ and $a^{\prime}$ are two orientations of analyzer 1 , and $b$ and $b^{\prime}$ are two orientations of analyzer 2 . There is nothing wrong with this, but then they use Equation (2) to obtain their famous inequality

$$
\begin{array}{r}
-1 \leq p_{12}(a, b)-p_{12}\left(a, b^{\prime}\right)+p_{12}\left(a^{\prime} b\right)+p_{12}\left(a^{\prime}, b^{\prime}\right) \\
-p_{1}\left(a^{\prime}\right)-p_{2}(b) \leq 0
\end{array}
$$


The use of this inequality to interpret the results of $f_{30}$ EPR-Bohm experiments is, for the reasons stated above, 331 meaningless.

\section{B. Wigner, Belinfante and Holt}

Clauser and Shimony[6], in Section 3.7 discuss 337 Bell Inequalities developed independently by Wigner[8], Belinfante[9], and Holt[10]. Their method consists of subdividing the space $\Lambda$ of states of a two-component system into subspaces corresponding to various possible values of ${ }^{338}$ the observables of interest, and then performing calculations on the measures of these subspaces. Clauser and ${ }^{339}$ Shimony show how their method can be used to derive ${ }^{340}$ the $\mathrm{CH}$ Inequality (Equation (5), above). That should ${ }^{341}$ be enough to disqualify them from being used for EPR-342 Bohm experiments, but it is instructive to see why they ${ }^{343}$ are equivalent, using Clauser and Shimony's analysis. ${ }^{344}$

They start with the assumption that the detection or ${ }^{345}$ non-detection of component 1 is completely determined ${ }^{346}$ by the parameter a of the first analyzer and the state of ${ }^{347}$ the composite system, but is independent of the parame-348 ter $\mathrm{b}$ of the other analyzer, and likewise for component $2 .{ }^{349}$ They then assume that determinism applies and exhaus-350 tively subdivide the space $\Lambda$ into 16 mutually disjoint ${ }^{351}$ subspaces $\Lambda(i j ; k l)$, where each letter can take the value ${ }^{352}$ 0 or 1 , with 1 denoting detection and 0 non-detection.;353 with $\mathrm{i}$ and $\mathrm{j}$ referring to the results if the parameter of ${ }_{354}$ the first analyzer is chosen respectively to be a or $a^{\prime}$; $\operatorname{and}_{355}$ with $\mathrm{k}$ and $\mathrm{l}$ referring to the results if the parameter of the second analyzer is chosen respectively to be $\mathrm{b}$ or $b^{\prime}$. If a probability measure $\rho$ is assumed to be given on $\Lambda$, then $\rho(i j ; k l)$ is defined to be the probability that the composite state is in $\Lambda(i j ; k l)$. All the $\rho(i j ; k l)$ are non ${ }^{356}$ negative, and because the 16 subspaces are disjoint and ${ }^{357}$ exhaustive, we have:

$$
\sum_{i j k l} \rho(i j ; k l)=1
$$

We now define $p_{1}(a)$ to be the probability that component 1 will be detected if the parameter is chosen to be a;359 $p_{2}(b)$ to be the probability that component 2 will be be 360 detected if its parameter is chosen to be b; and $p_{12}(a, b)$ to be the probability of joint detection of both components if the two parameters are chosen respectively to be $\mathrm{a}$ and $\mathrm{b}$. Analogous definitions are given for the values of the other parameters. Then, for example, $p_{12}(a, b)=\rho(11 ; 11)+\rho(11 ; 10)+\rho(10 ; 11)+\rho(10 ; 10)^{363}$ $p_{12}\left(a, b^{\prime}\right)=\rho(11 ; 11)+\rho(11 ; 01)+\rho(10 ; 11)+\rho(10 ; 01){ }_{366}^{365}$ etc., and, for example,

$$
\begin{array}{r}
p_{2}(b)=\rho(11 ; 11)+\rho(11 ; 10)+\rho(10 ; 11)+\rho(10 ; 10) \\
+\rho(01 ; 11)+\rho(01 ; 10)+\rho(00 ; 11)+\rho(00 ; 10)
\end{array}
$$

Using these, and equivalent equations for the other probabilities, Clauser and Shimony show that Equation (5), above, is recovered. This deterministic approach, because of its disjoint subspaces, also does not allow for correlation between the particles; so it is not surprising that it is equivalent to the $\mathrm{CH}$ Inequality, a fact that is supported by Fine[11] (see Section II.F below), and, thus is inappropriate for EPR-Bohm experiments

\section{Bell}

The concept of Bell Inequalities was introduced by Bell[3] in 1964. Bell's paper dealt with an EPR-Bohm experiment using fermions in a singlet state moving in opposite directions. Measurements are made by SternGerlach magnets on selected components of the spins $\vec{\sigma}_{1}$ and $\vec{\sigma}_{2}$. If measurement of the component $\vec{\sigma}_{1} \cdot \vec{a}$ where $\vec{a}$ is some unit vector, yields the value -1 then, according to quantum mechanics, measurement of $\vec{\sigma}_{2} \cdot \vec{a}$ must yield the value -1 and vice versa. Since this is true even when the measurements are made at places remote from one another, it implies the possibility of a more complete specification of the state, as proposed by EPR, which he denotes by $\lambda$, where $\lambda$ is quite general, denoting whatever is needed for a complete specification.

The result $A$ of measuring $\vec{\sigma}_{1} \cdot \vec{a}$ is then determined by $\vec{a}$ and $\lambda$, and the result $B$ of measuring $\vec{\sigma}_{2} \cdot \vec{b}$ in the same instance is determined by $\vec{b}$ and $\lambda$; so

$$
A(\vec{a}, \lambda)= \pm 1 \text { and } B(\vec{b}, \lambda)= \pm 1
$$

Then he states that if $\rho(\lambda)$ is the probability distribution of $\lambda$, the expectation value of the product of the two components $\vec{\sigma}_{1} \cdot \vec{a}$ and $\vec{\sigma}_{2} \cdot \vec{b}$ is

$$
P(\vec{a}, \vec{b})=\int d \lambda \rho(\lambda) A(\vec{a}, \lambda) B(\vec{b}, \lambda)
$$

which he states is equivalent to the quantum mechanical expectation value

$$
<\vec{\sigma}_{1} \cdot \vec{a} \vec{\sigma}_{2} \cdot \vec{b}>=-\vec{a} \cdot \vec{b}
$$

The factorization in Equation (7), as pointed out by Clauser and Shimony in their Section 3.1 Equation (3.4), is an expression of locality for a deterministic hiddenvariables theory. As discussed in Section A, above, it is not an acceptable locality condition.

Thus, there is no need to discuss Bell's development further, because the inequalities are inappropriate, are never used anyway and the development contains some dubious steps. In 1971 Bell[12] offered an updated and improved proof, but it contains the same factorization as Equation (7) and thus also is inappropriate. 


\section{Stapp}

In a paper describing the "S-Matrix Interpretation of ${ }^{427}$ Quantum Theory" Stapp[13] discusses the completeness ${ }^{428}$ of quantum theory with the use of an EPR-Bohm experiment. Two fermions in a singlet spin state, traveling in opposite directions, each pass through a Stern-Gerlach ${ }^{429}$ type device. The axes of the two devices $\left(A_{1}\right.$ and $\left.A_{2}\right)$ are denoted by $D_{1}$ and $D_{2}$. They are both normal to ${ }^{430}$ the c.m. line of flight, and $\theta\left(D_{1}, D_{2}\right)$ is the angle between them. Two different settings, $D_{1}^{\prime}$ and $D_{1}^{\prime \prime}$ of $D_{1431}$ are considered; and two different settings, $D_{2}^{\prime}$ and $D_{2}^{\prime \prime}$ of ${ }_{432}$ $D_{2}$ are considered. Altogether four alternative combina-433 tions of settings are considered. Let $\mathrm{j}$ label the individ- ${ }_{434}$ ual experiment, i.e., a single pair of particles in a singlet 435 state. Then let $n_{1 j}\left(D_{1}, D_{2}\right)$ be defined to be +1 or $-1,436$ according to whether the theory predicts that the parti-437 cle from the $j t h$ pair that passes through $A_{1}$ is deflected ${ }_{438}$ up or down, when the settings of the axes are $D_{1}$ and $D_{2.439}$ The numbers $n_{2 j}\left(D_{1}, D_{2}\right)$ are defined analogously for the ${ }_{440}$ other particle. Based on the condition that the theory ${ }_{441}$ gives predictions for various possible alternative settings, for each individual pair $\mathrm{j}$, the numbers $n_{i j}\left(D_{1}, D_{2}\right)$ and $n_{2 j}\left(D_{1}, D_{2}\right)$ are defined for all four of the arguments $D_{1}$ and $D_{2}$.

Stapp then discusses the fact that, based on his pre- 442 dictability condition, the eight numbers $n_{i j}\left(D_{1}, D_{2}\right)$ are 443 defined for each experiment $j$. Of these eight num-444 bers only two can be compared to experiment. The 445 other six correspond to the three alternative experiments446 that could have been performed but were not. He then ${ }_{447}$ states that if the experimenters had actually adjusted the mechanical devices to give the alternative experimental setup, then the alternative experiments would ${ }_{448}$ have had certain definite results. Then the numbers $n_{1 j}\left(D_{1}, D_{2}\right)$ and $n_{2 j}\left(D_{1}, D_{2}\right)$ can be defined by the results that the experiments would have had if they had ac- ${ }^{449}$ tually been performed. (This is what is called "counterfactual definiteness" and Eberhard[14] refers to as "conjugate events".) This is an interesting concept and poten- ${ }^{45}$ tially could be used to introduce correlation into the theory, because there is a correlation between $n_{1 j}\left(D_{1}, D_{2}\right)$ and $n_{2 j}\left(D_{1}, D_{2}\right)$ for each j. It's not clear how to do that, ${ }_{451}$ and there is no indication that Stapp attempted to do so.

Stapp then states that the following relationship with quantum mechanics should hold with increasing accuracy as $\mathrm{N}$ increases:

$$
\frac{1}{N} \sum_{j=1}^{N} n_{1 j}\left(D_{1}, D_{2}\right) n_{2 j}\left(D_{1}, D_{2}\right)=-\cos \theta\left(D_{1}, D_{2}\right)
$$

It is clear that this suffers from the same factorization problem of Equation (1) in Section II.A, above. No provision is made for a correlated $n_{12 j}\left(D_{1}, D_{2}\right)$. Because there is no correlation between the particles, the method ${ }^{453}$ is meaningless for the interpretation of EPR-Bohm experiments. Clauser and Shimony point out that in 1978 Stapp[15] responded to some critics of his earlier paper 454 and also provided an equivalence theorem that shows his approach is, in fact, equivalent to Equation (2) in Section II.A, above, confirming that it is an inappropriate measure.

\section{E. Eberhard}

\section{Eberhard 1977}

In 1977 Eberhard[16] published a paper purporting to derive Bell Inequalities without using the concept of hidden variables. He considers an EPR-Bohm experiment with two measuring locations, $\mathrm{A}$ and $\mathrm{B}$, at a distance from each other, with a knob, a, on apparatus $\mathrm{A}$ and a knob, b, on apparatus B. N events are recorded, with a measurement on both A and B. There are only two outcomes for each measurement, +1 or -1 . The $j t h$ event corresponds to a response $\alpha_{j}$ in $\mathrm{A}$ and $\beta_{j}$ in B. Each term $\alpha_{j}$ and $\beta_{j}$ is either +1 or -1 . He defines correlation by a statistical mean

$$
C=\frac{1}{N} \sum_{j} \alpha_{j} \beta_{j}=\left\langle\alpha_{j} \beta_{j}>\right.
$$

where the brackets around a quantity designates the statistical mean of that quantity over $\mathrm{j}$. $\mathrm{C}$ is equal to the fraction of events when $\alpha_{j}$ and $\beta_{j}$ have the same signs minus the fraction where they have opposite signs. There are two positions $a^{(1)}$ and $a^{(2)}$ of the knob a and two positions, $b^{(1)}$ and $b^{(2)}$ for the knob b. He defines:

$$
\begin{aligned}
& C^{(1,1)}=<\alpha_{j} \beta_{j}>, a=a^{(1)}, b=b^{(1)} \\
& C^{(2,1)}=<\alpha_{j} \beta_{j}>, a=a^{(2)}, b=b^{(1)} \\
& C^{(1,2)}=<\alpha_{j} \beta_{j}>, a=a^{(1)}, b=b^{(2)} \\
& C^{(2,2)}=<\alpha_{i} \mid \beta_{j}>, a=a^{(2)}, b=b^{(2)}
\end{aligned}
$$

and then defines for event $\mathrm{j}$ :

$$
\gamma_{j}=\alpha_{j}^{(1)} \beta_{j}^{(1)}+\alpha_{j}^{(2)} \beta_{j}^{(1)}+\alpha_{j}^{(1)} \beta_{j}^{(2)}-\alpha_{j}^{(2)} \beta_{j}^{(2)}
$$

Each product $\alpha_{j} \beta_{j}$ is equal to \pm 1 ; so therefore

$$
\begin{aligned}
\frac{1}{N} \sum_{j} \gamma_{j}=<\gamma & =<\alpha_{j}^{(1)} \beta_{j}^{(1)}>+<\alpha_{j}^{(2)} \beta_{j}^{(1)}> \\
+ & <\alpha_{j}^{(1)} \beta_{J}^{(2)}>-<\alpha_{j}^{(2)} \beta_{j}^{(2)}>\leq 2
\end{aligned}
$$

and finally

$$
C^{(1,1)}+C^{(2,1)}+C^{(1,2)}-C^{(2,2)} \leq 2
$$

which is the famous CHSH[17] inequality. 
[It should be noted that the CHSH Inequality is obtained using a "correlation function", $P(a, b)=\sum_{\Gamma} A(a, \lambda) B(b, \lambda) \rho(\lambda) d \lambda$ which is a factored form that actually denies correlation of the particles. They don't even refer to a quantity like $A B(a, b, \lambda)$; so, clearly the CHSH Inequality suffers the same problem as the CH Inequality discussed in Section II.A, above, and is inappropriate for EPR-Bohm experiments.]

The statistical means used in the development by Eber- ${ }_{519}$ hard also do not provide for correlation between the par- ${ }_{520}$ ticles, which is why he reaches the same inappropriate ${ }_{521}$ inequality.

\section{Eberhard 1992}

In 1992 Eberhard[14] presented an alternative inequal- ${ }^{526}$ ity based on an entirely different approach. The initial state is prepared as a superposition of two photons a and $\mathrm{b}$, with correlated planes of polarization. One state is defined with photon a polarized vertically and photon b polarized horizontally while the other state has photon $\mathrm{a}^{527}$ polarized horizontally and photon $b$ vertically. The an-528 gle of the detection device for photon a is denoted $\alpha$ and ${ }^{529}$ that of photon $\mathrm{b}$ as $\beta$.

The Bell Inequalities are concerned with expectation ${ }^{531}$ values of quantities that can be measured in four different ${ }^{532}$ experimental setups defined by specific values $\alpha_{1}, \alpha_{2}, \beta_{1}{ }^{53}$ and $\beta_{2}$ of $\alpha$ and $\beta$. The setups are referred to by the symbols $\left(\alpha_{1}, \beta_{1}\right),\left(\alpha_{1}, \beta_{2}\right),\left(\alpha_{2}, \beta_{1}\right)$, and $\left(\alpha_{2}, \beta_{2}\right)$, where the first index designates the value of $\alpha$ and the second index the value of $\beta$.

In each setup, the "fate" of photon a and the "fate" of photon $b$ is referred to by an index (o) for photons de- ${ }^{534}$ tected in the ordinary beam, (e) for photons detected in the extraordinary beam, or $(\mathrm{u})$ for photons undetected.. Therefore there are nine types of events: $(\mathrm{o}, \mathrm{o}),(\mathrm{o}, \mathrm{u})$, $(\mathrm{o}, \mathrm{e}),(\mathrm{u}, \mathrm{o}),(\mathrm{u}, \mathrm{u})(\mathrm{u}, \mathrm{e}),(\mathrm{e}, \mathrm{o}),(\mathrm{e}, \mathrm{u})$ and $(\mathrm{e}, \mathrm{e})$, where the first index designates the fate of photon a and the second index the fate of photon b. He defines conditions:

(i) The fate of photon a is independent of the value of $\beta, 536$ i.e., is the same in an event of the sequence corresponding ${ }^{537}$ to setup $\left(\alpha_{1}, \beta_{1}\right)$ as in the event with the same event538 number $\mathrm{k}$ for $\left(\alpha_{1}, \beta_{2}\right)$; also same fate for $\mathrm{a}$ in $\left(\alpha_{2}, \beta_{1}\right)^{539}$ and $\left(\alpha_{2}, \beta_{2}\right)$; this is true for each $\mathrm{k}$ for these sequences. ${ }^{540}$

(ii) The fate of photon $\mathrm{b}$ is independent of the value of ${ }^{541}$ $\alpha$, i.e., is the same in event $\mathrm{k}$ for sequences $\left(\alpha_{1}, \beta_{1}\right)$ and $\mathrm{d}^{542}$ $\left(\alpha_{2}, \beta_{1}\right)$; also same fate for $\mathrm{b}$ in sequences $\left(\alpha_{1}, \beta_{2}\right)$ and $\left(\alpha_{2}, \beta_{2}\right)$.

These are perfectly reasonable locality conditions, but ${ }^{543}$ they only apply for any given $\mathrm{k}$, not for the sums over all $\mathrm{k}$.

Following an argument first used by Stapp[13], when ${ }_{545}$ four sequences are found satisfying conditions (i) and (ii),546 the four events with the same event number $\mathrm{k}$ in the four 547 sequences are called "conjugate events". He then uses the notation $n_{f_{1} f_{2}}(\alpha, \beta)$, where $f_{1}$ is the fate (o,e,or $\left.\mathrm{u}\right)$ of photon $a$ and $f_{2}$ is the fate of photon $b$, for the number of events with that outcome. For example, $n_{o o}\left(\alpha_{1}, \beta_{1}\right)$ is the number of events with the outcome o for photon $a$ and with the outcome o for photon $b$ when $\alpha$ is $\alpha_{1}$ and $\beta$ is $\beta_{1}$.

[Note: This is the number of events with that outcome, not an event for a single value of $\mathrm{k}$, and that the concept of "conjugate event" does not really apply. Nevertheless, Eberhard proceeds to develop his inequality using these totals, which for notational purposes he calls boxes.]

He starts by considering $n_{o o}\left(\alpha_{1}, \beta_{1}\right)$. He then subtracts from that total some of the boxes for which events, had they been for the same $\mathrm{k}$, would have been conjugate to events that go into the box $n_{o o}\left(\alpha_{1}, \beta_{1}\right)$.

$$
\begin{aligned}
n_{\text {oo }}\left(\alpha_{1}, \beta_{1}\right) & -n_{\text {uo }}\left(\alpha_{2}, \beta_{1}\right)-n_{e o}\left(\alpha_{2}, \beta_{1}\right) \\
& -n_{\text {ou }}\left(\alpha_{1}, \beta_{2}\right)-n_{\text {oe }}\left(\alpha_{1}, \beta_{2}\right)
\end{aligned}
$$

It's not immediately clear why he did not also consider the boxes, $n_{o o}\left(\alpha_{2}, \beta_{1}\right)$ and $n_{o o}\left(\alpha_{1}, \beta_{2}\right)$, which also meet the conditions (i) and (ii), though they are both conjugate to the box that he then includes, $n_{o o}\left(\alpha_{2} \beta_{2}\right)$. Because the remainder shown in Expression(12), above must be less than or equal to the number in box $n_{o o}\left(\alpha_{2}, \beta_{2}\right)$, he obtains:

$$
\begin{aligned}
n_{\text {oo }}\left(\alpha_{1}, \beta_{1}\right)-n_{u o}\left(\alpha_{2}, \beta_{1}\right) & -n_{e o}\left(\alpha_{2}, \beta_{1}\right)-n_{\text {ou }}\left(\alpha_{1}, \beta_{2}\right) \\
& -n_{o e}\left(\alpha_{1}, \beta_{2}\right) \leq n_{o o}\left(\alpha_{2}, \beta_{2}\right)
\end{aligned}
$$

, or his inequality:

$$
\begin{array}{r}
n_{\text {oe }}\left(\alpha_{1}, \beta_{2}\right)+n_{\text {ou }}\left(\alpha_{1}, \beta_{2}\right)+n_{\text {eo }}\left(\alpha_{2}, \beta_{1}\right)+n_{\text {uo }}\left(\alpha_{2}, \beta_{1}\right) \\
+n_{\text {oo }}\left(\alpha_{2}, \beta_{2}\right)-n_{\text {oo }}\left(\alpha_{1} \cdot \beta_{1}\right) \geq 0
\end{array}
$$

He then claims that Equation (14) is equivalent to Equation (5) of Section II.A, above. He also points out that if the o's and e's in Equation (14) are interchanged one obtains a similar inequality, which, if averaged with Equation (14) yields an inequality essentially identical to the CHSH Inequality mentioned in Section II.E.1,above. Clearly, this makes his inequality inappropriate for EPRBohm experiments for the reasons discussed above.

\section{Giustina et.al}

Another form of Eberhard's inequality was developed by Giustina, et.al.[18]. In an experiment, one records measurements of singles counts, $S$ (number of detection events on one side), and $\mathrm{C}$ (number of 
detected pairs), for the four combination of settings587 $\left(\alpha_{1}, \beta_{1}\right),\left(\alpha_{1}, \beta_{2}\right),\left(\alpha_{2}, \beta_{1}\right)$ and $\left(\alpha_{2}, \beta_{2}\right)$.

The number of events for which one of the outcomes isso undetected follows from the measured rates. For a given measurement length the measured coincident counts are $C_{k l}\left(\alpha_{i}, \beta_{j}\right)$ and the single counts are $S_{k}^{\alpha}\left(\alpha_{i}\right)$ and $S_{l}^{\beta}\left(\beta_{j}\right)$, where $\mathrm{k}$ and $\mathrm{l}$ are o or e. The terms in Eberhard's inequality, Equation (14) above, are then given by the following ${ }^{590}$ measured quantities:

$$
n_{o o}\left(\alpha_{1}, \beta_{1}\right)=C_{o o}\left(\alpha_{1}, \beta_{1}\right)
$$

$$
n_{o e}\left(\alpha_{1}, \beta_{2}\right)=C_{o e}\left(\alpha_{1}, \beta_{2}\right)
$$

$$
n_{\text {ou }}\left(\alpha_{1}, \beta_{2}\right)=S_{o}^{\alpha}\left(\alpha_{1}\right)-C_{\text {oo }}\left(\alpha_{1}, \beta_{2}\right)-C_{o e}\left(\alpha_{1} \beta_{2}\right)
$$

$$
n_{e o}\left(\alpha_{2}, \beta_{1}\right)=C_{e o}\left(\alpha_{2}, \beta_{1}\right)
$$

$$
n_{u o}\left(\alpha_{2}, \beta_{1}\right)=S_{o}^{\beta}\left(\beta_{1}\right)-C_{o o}\left(\alpha_{2}, \beta_{1}\right)-C_{e o}\left(\alpha_{2}, \beta_{1}\right)
$$

$$
n_{\text {oo }}\left(\alpha_{2}, \beta_{2}\right)=C_{\text {oo }}\left(\alpha_{2}, \beta_{2}\right)
$$

Inserting these expressions into Equation (14) above $_{600}$ yields:

$$
\begin{array}{r}
-C_{o o}\left(\alpha_{1}, \beta_{1}\right)+S_{o}^{\alpha}\left(\alpha_{!}\right)-C_{o o}\left(\alpha_{1}, \beta_{2}\right) \\
+S_{o}^{\beta}\left(\beta_{1}\right)-C_{o o}\left(\alpha_{2}, \beta_{1}\right)+C_{o o}\left(\alpha_{2}, \beta_{2}\right) \geq 0
\end{array}
$$

,where the coincidence counts $C_{o e}\left(\alpha_{1}, \beta_{2}\right)$ and $_{612}$ $C_{e o}\left(\alpha_{2}, \beta_{1}\right)$ have dropped out. The resulting in-613 equality now contains only directly available detection ${ }_{614}$ events related to the ordinary beams of $\alpha$ and $\beta$. This ${ }_{615}$ inequality was used by Giustina, et. al., in their exper-616 iment and was used in the recent NIST experiment[4].617 Obviously it suffers from the same lack of correlation $_{618}$ as the Eberhard[14] inequality above, upon which it is ${ }_{619}$ based.

\section{F. Summary}

The list of Bell Inequalities discussed above is not625 exhaustive and each is not dealt with rigorously, just 626 enough to give a flavor of the development and to show 627 that it does not provide for correlation between the cor-628 related particles. It also shows that it does not matter629 whether a deterministic hidden-variables model or a fac-630 torized stochastic model is used. They all preclude the6з1 correlation between the particles.

This is not surprising in light of a 1982 paper by633 Fine[11] which proves that every deterministic hidden-634 variables model is equivalent to a factorizable stochas-635 tic model. (See also Appendix A of Hall[19].) It is the 636 factorized probability that makes these models unsuited,637 even irrelevant, for use in interpreting the results of EPRBohm experiments. The probability for the factorized model

$$
p_{12}(a, b)=\int_{\Lambda} d \lambda \rho(\lambda) p_{1}(\lambda, a) p_{2}(\lambda, b)
$$

correctly accounts for the locality of the measuring devices, but ignores the fact that each pair of particles is correlated and that the measurements for each pair must correspondingly be correlated. (This is true whether one proposes, as the EPR view does, that the spin/polarization of the particles have simultaneous reality or whether, instead, one contends that the spin/polarization of the particles only are realized at the time of the measurements.) For the EPRBohm experiments, $p_{12}(\lambda, a, b)$ is definitely not equal to $p_{1}(\lambda, a) p_{2}(\lambda, b)$. Thus one must conclude, for the reasons expressed in the discussion of Equation(1) and Equation(2) of Section II.A above, that the Bell Inequalities are inappropriate for use in interpreting the results of EPR-Bohm experiments.

\section{CONCLUSION}

It is shown that none of the Bell Inequalities have any relevance for EPR-Bohm experiments. The extreme locality conditions they use do not account for, or even allow consideration of, the correlation between pairs of particles; but it is the correlation between a pair of particles that is the characteristic feature of such experiments. The only locality condition required by EPR is that a measurement on one of the particles is not affected by and can not affect the measurement made on the second particle. There is therefore no reason why in an EPR-Bohm experiment that the inequalities should not be violated; in fact one should expect that they would be violated, because they express conditions that are decidedly untrue if the experimenters did their jobs well.

In light of this it would be incorrect to claim that violation of Bell Inequalities requires the conclusion that the measurement of one particle in an "entangled" pair can instantly affect the state of its partner, even at a great distance. This action at a distance may be true, but that is not proved by the results of any EPR-Bohm experiments yet performed. It's also possible that Einstein, Podolsky and Rosen are correct in claiming that there is simultaneous reality for the quantities being measured and thus quantum mechanics provides an incomplete description.

Because the experiments have been performed at space-like separations, there are really two possible interpretations: either

(1) the spin/polarization state of each particle has simultaneous reality, and a measurement on one, though related to, does not affect a measurement on the other, or 
(2) contrary to all of our hard-earned current under-643 standing of space-time, the spin/polarization state of each particle only becomes real at the time of measure-644 ment and the measurement of one immediately deter-645 mines the state of the other, even when they are space-646 like separated.

Although each interpretation is, perhaps, possible, it would be irresponsible to proclaim that violation of Bell Inequalities requires the second.
[1] A. Einstein, B. Podolsky, and N. Rosen, Phys. Rev. 47,667 777 (1935).

668

[2] D. Bohm and Y. Aharonov, Phys. Rev. 108, 1070 (1957).669

[3] J. S. Bell, Physics 1, 195 (1964).

\section{0}

4] A. Aspect, P.Grangier, and G. Roger, Phys. Rev. Lett.671 49, 91 (1982); A. Aspect, J. Dalibard, and G. Roger, 672 ibid. 49, 1804 (1982); L. K. Shalm, E. Meyer-Scott, B. G.673 Christensen, P. Bierhorst, M. A. Wayne, M. J. Stevens,674 T. Gerrits, S. Glancy, D. R. Hamel, M. S. Allman, et al.,675 ibid. 115, 250402 (2015).

[5] D. J. Griffiths, Introduction to Quantum Mechan-677 ics (Benjamin Cummings, 2004); B. Greene,678 The Fabric of the Cosmos (Vintage Books, 2005);679 D. I. Kaiser, How the Hippies Saved Physics (W.680 W. Norton, 2011); L. Ost, NIST Team Proves681 'Spooky Action at a Distance' is Real (2015), url-682 http://www.nist.gov/pml/div686/20151105loophole.cfm\$\$83 D. Powell, Discover 37, 58 (2016).

[6] J. F. Clauser and A. Shimony, Rep. Prog. Phys. 41, $1881_{685}$ (1978).
[7] J. F. Clauser and M. A. Horne, Phys. Rev. D 10, 526 (1974).

[8] E. P. Wigner, Am. J. Phys. 38, 1005 (1970).

[9] F. J. Belinfante, A Survey of Hidden-Variables Theories (Oxford: Pergamon, 1973).

[10] R. A. Holt, Atomic Cascade Experiments, Ph.D. thesis, Harvard University (1973).

[11] A. Fine, Phy. Rev. Lett. 48, 291 (1982).

[12] J. S. Bell, Foundations of Quantum Mechanics (New York: Academic, 1971) pp. 171-81.

[13] H. P. Stapp, Phys. Rev. D 3, 1303 (1970).

[14] P. H. Eberhard, Phys. Rev. A 47, R747 (1993).

[15] H. P. Stapp, Found. of Phys. 9, 1 (1979).

[16] P. H. Eberhard, Nuovo Cim. 38B, 75 (1977).

[17] J. F. Clauser, M. A. Horne, A. Shimony, and R. A. Holt, Phys. Rev. Lett. 23, 880 (1969).

[18] M. Giustina, A. Mech, S. Ramelow, B. Wittmann, J. Kofler, J. Beyer, A. Lita, B. Calkins, T. Gerrits, et al., Nature 497, 227 (2013).

[19] M. J. W. Hall, Phys. Rev. A 84, 022102 (2011). 